\title{
Opto-mechanical frequency shifting of scattered light
}

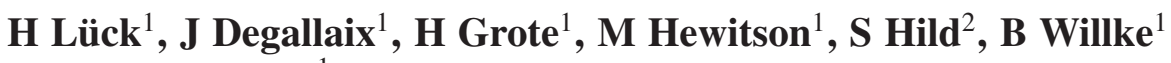 \\ and K Danzmann ${ }^{1}$ \\ ${ }^{1}$ Max-Planck-Institut für Gravitationsphysik (Albert-Einstein-Institut) and Leibniz Universität \\ Hannover, Callinstrasse 38, D-30167 Hannover, Germany \\ ${ }^{2}$ School of Physics and Astronomy, University of Birmingham, Edgbaston, \\ Birmingham B15 2TT, UK \\ E-mail: harald.lueck@aei.mpg.de
}

Received 4 April 2008, accepted for publication 27 June 2008

Published 21 July 2008

Online at stacks.iop.org/JOptA/10/085004

\begin{abstract}
Light being scattered back from external surfaces or optical components can affect the sensitivity of optical measurement systems such as interferometers. By modulating the optical path length in front of a critical optical component the noise contributions due to the scattered light can be shifted out of the frequency band of interest. An opto-mechanical phase shifter has been designed for this purpose and noise reduction has been demonstrated.
\end{abstract}

Keywords: scattering, modulation, interferometer, gravitational waves

(Some figures in this article are in colour only in the electronic version)

\section{Introduction}

Scattered light often poses great problems in reaching the sensitivity goals of optical experiments. In the current laser-interferometric gravitational-wave detectors (LIGO [1], TAMA300 [2], Virgo [3] and GEO 600 [4]), a fraction of the order of $10^{-23} \mathrm{~Hz}^{-1 / 2}$ of the circulating light power being scattered back into the interferometer mode is sufficient to spoil the sensitivity [5]. Often it is very hard to avoid backscattering because some components, e.g. radio-frequency photodiodes, may need to be placed close to small beam waists, which maximizes the amount of light being scattered back into the main beam path [5]. In this case the device described in this paper can frequency shift the scatter noise without adverse unwanted consequences. For locating limiting scatter sources it can easily be inserted into an existing setup without the need to modify any optical elements. We will use an interferometric gravitational-wave detector to describe the operational principle but the method can also be used in many other optical arrangements where scattered light becomes a problem. The effect exploited has briefly been described in another context [7] and is explained in detail in this paper.

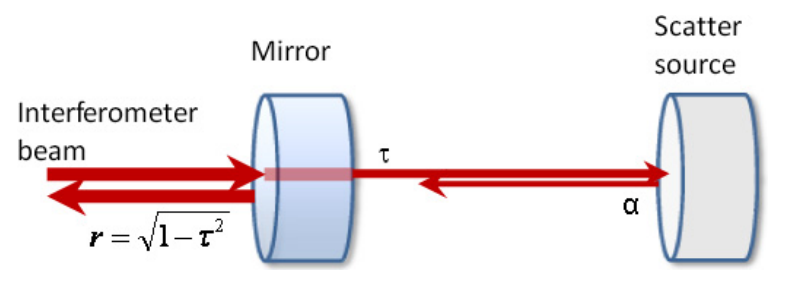

Figure 1. Scattering source behind a mirror of the interferometer.

\section{Effect of scattered light}

Figure 1 shows a scenario in which the main laser beam in an interferometer arm is partly transmitted through a mirror (with an amplitude transmission coefficient of $\tau$ ) and then hits a scattering surface. A small fraction of the scattered light will pass back through the mirror into the same direction as the main laser beam and will interfere with it.

If the component scatters a fraction $\alpha$ of the incident light and moves with an amplitude $a(t)$ with respect to the rest of the instrument, this will cause a phase modulation of the scattered light, by $\phi=2 a(t) 2 \pi / \lambda$. If the initial beam inside the interferometer is described by a field

$$
E_{0}(t)=E_{0} \mathrm{e}^{\mathrm{i} \omega_{0} t}
$$




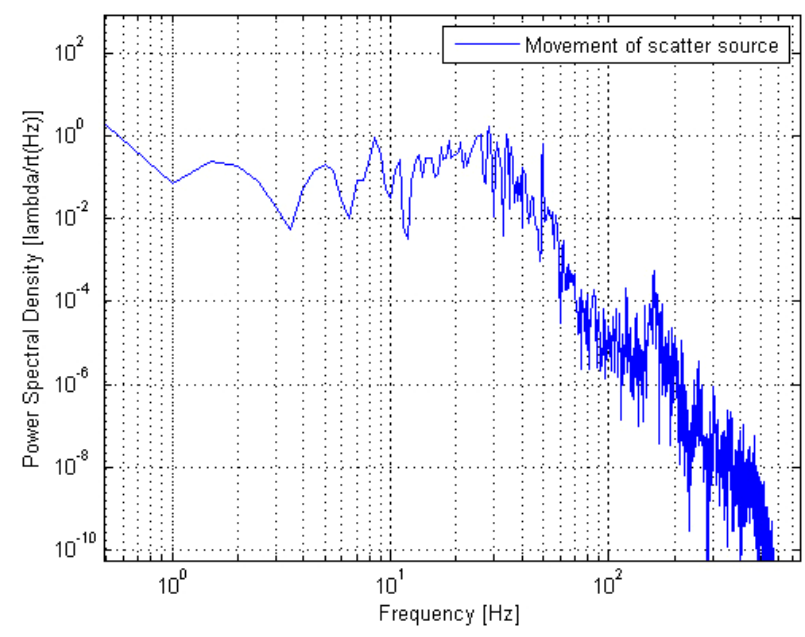

Figure 2. Example spectrum for the movement of the scattering source along the optical path. The spectrum shown is an arbitrary recorded seismic signal scaled to yield a strong scattering signal in the most sensitive frequency band of ground-based gravitational-wave detectors.

then the modulated field after reflection from the scatterer will be

$$
E_{\mathrm{m}}(t)=\alpha \tau E_{0} \mathrm{e}^{\mathrm{i}\left(\omega_{0} t+\frac{2 \pi}{\lambda} 2 a(t)\right)} .
$$

The phase-modulated beam returning from the scatter source (transmitted again through the mirror) will interfere with the main beam with a matching efficiency $\epsilon$, yielding the field

$$
E_{\text {beat }}(t)=r E_{0} \mathrm{e}^{\mathrm{i} \omega_{0} t}+\tau^{2} \alpha \epsilon E_{0} \mathrm{e}^{\mathrm{i}\left(\omega_{0} t+\frac{4 \pi}{\lambda} a(t)\right)}
$$

where $r=\sqrt{\left(1-\tau^{2}\right)}$ is the reflectivity of the mirror. On a photodiode this gives the detected intensity

$$
\begin{aligned}
& I_{\text {beat }}(t) Z_{0}=\left|E_{\text {beat }}(t)\right|^{2} \\
& \quad=\text { const }+r \tau^{2} \alpha \in I_{0}\left[\mathrm{e}^{\mathrm{i} \frac{4 \pi}{\lambda} a(t)}+\mathrm{e}^{-\mathrm{i} \frac{4 \pi}{\lambda} a(t)}\right]
\end{aligned}
$$

with $I_{0}=E_{0}^{2} / Z_{0}$, with $Z_{0}=\sqrt{\mu_{o} / \epsilon_{0}}$ the characteristic impedance of a vacuum.

The Fourier transform of the intensity, representing the signal content at the frequency $\omega=2 \pi f$ (disregarding the constant term), is given by

$$
\begin{aligned}
& X(\omega)=\frac{1}{\sqrt{2 \pi}} \int_{-\infty}^{\infty} I_{\text {beat }}(t) \mathrm{e}^{\mathrm{i} \omega t} \mathrm{~d} t \\
& =\frac{r \tau^{2} \alpha \epsilon I_{0}}{\sqrt{2 \pi}} \int_{-\infty}^{\infty}\left(\mathrm{e}^{\mathrm{i} \frac{4 \pi}{\lambda} a(t)}+\mathrm{e}^{-\mathrm{i} \frac{4 \pi}{\lambda} a(t)}\right) \mathrm{e}^{\mathrm{i} \omega t} \mathrm{~d} t \\
& =\underbrace{\frac{r \tau^{2} \alpha \epsilon I_{0}}{\sqrt{2 \pi}} \int_{-\infty}^{\infty} \mathrm{e}^{\mathrm{i} \frac{4 \pi}{\lambda} a(t)} \mathrm{e}^{\mathrm{i} \omega t} \mathrm{~d} t}_{X^{+}(\omega)} \\
& +\underbrace{\frac{r \tau^{2} \alpha \epsilon I_{0}}{\sqrt{2 \pi}} \int_{-\infty}^{\infty} \mathrm{e}^{-\mathrm{i} \frac{4 \pi}{\lambda} a(t)} \mathrm{e}^{\mathrm{i} \omega t} \mathrm{~d} t}_{X^{-}(\omega)} .
\end{aligned}
$$

We should note here that the Fourier transform $\mathcal{F}(\omega)$ of a signal $S(t)$ multiplied by $\mathrm{e}^{\mathrm{i} \Omega t}$ just shifts the Fourier transform by $\Omega$ :

$$
\mathcal{F}\{S\}(\omega) \mathrm{e}^{\mathrm{i} \Omega t}=\mathcal{F}\{S\}(\omega+\Omega) .
$$

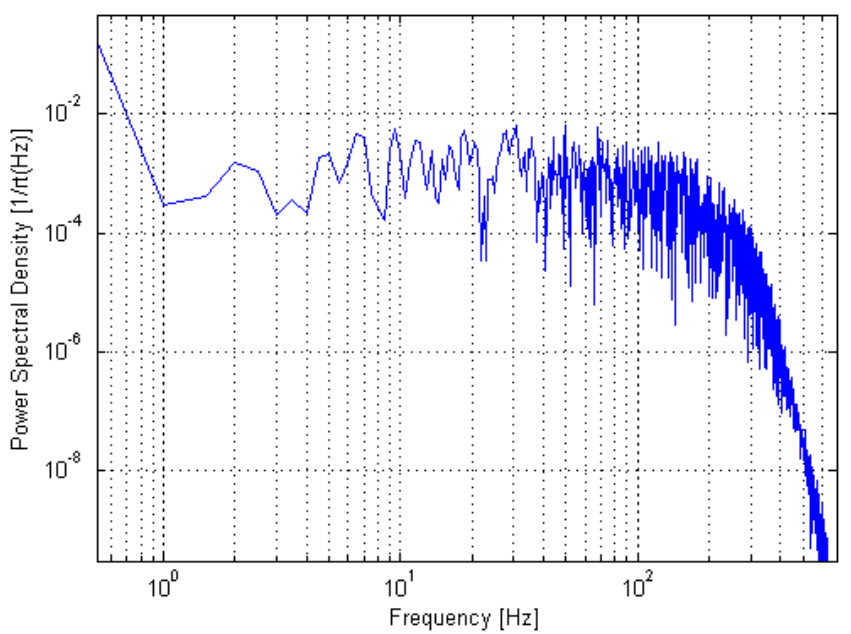

Figure 3. Simulated resulting noise spectrum on the returning interferometer beam with a movement of the scatterer as assumed in figure 2. For this example $\tau^{2}=0.6$ and $\alpha \epsilon=1$, i.e. close to maximum 'scatter efficiency', has been assumed. Comparing figures 2 and 3 shows that the assumed movement produces a typical 'scattering shoulder' with a flat spectrum up to a corner frequency (about $100 \mathrm{~Hz}$ in this case) above which the spectral content rapidly falls off.

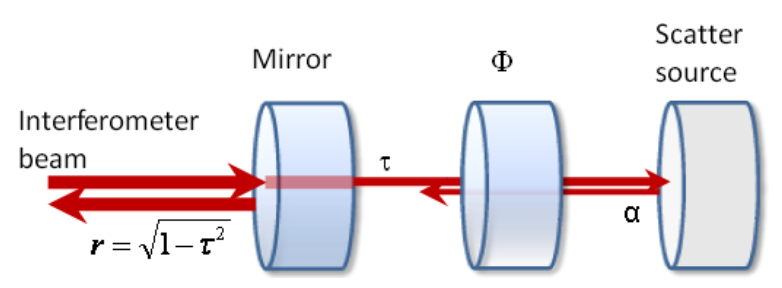

Figure 4. Phase shifter inserted between a mirror of the interferometer and the scattering source.

An example spectrum of the movement of a scattering source and the simulated resulting scattering noise is shown in figures 2 and 3, respectively.

\section{Frequency shifting the scattered light}

Figure 4 shows a phase modulator inserted between the mirror and the scattering source of figure 1. This allows phase modulation of the returning light interfering with the main laser beam inside the interferometer, and equation (3) turns into

$$
E_{\text {beat }}^{\mathrm{m}}(t)=r E_{0} \mathrm{e}^{\mathrm{i} \omega_{0} t}+\mathrm{e}^{\mathrm{i} M \cos \left(\Omega_{\mathrm{m}} t\right)} \tau^{2} \alpha \epsilon E_{0} \mathrm{e}^{\mathrm{i}\left(\omega_{0} t+\frac{4 \pi}{\lambda} a(t)\right)}
$$

where $M$ is the modulation index and $\Omega_{\mathrm{m}}$ the modulation frequency. By placing a phase shifter in the beam path we not only modulate the beam returning towards the mirror but also the incoming beam. The final (scattered and modulated) beam being overlapped with our main beam now has seen three subsequent phase modulations: from the modulator $\Phi_{1}(t)$, from the scattering source $\Phi_{2}(t)$ and from the modulator $\Phi_{3}(t)=\Phi_{1}(t)$ again. At first glance it may appear as a problem that one of the modulations has happened before the scattering process and the other one afterwards. If the 
delay time between the subsequent phase modulations is short compared with a full modulation period the phase modulations are commutative. This can easily be seen in the time domain. Whether the beam experiences $\Phi_{1}\left(t_{1}\right)+\Phi_{2}\left(t_{2}\right)+\Phi_{3}\left(t_{3}\right)$ (where $t_{1}, t_{2}$ and $t_{3}$ are the times the beam passes through the respective phase modulation stages) or $\Phi_{2}\left(t_{1}\right)+\Phi_{1}\left(t_{2}\right)+\Phi_{3}\left(t_{3}\right)$ does not matter. The sum, i.e. the overall phase change, is the same in both cases if $t_{1} \simeq t_{2} \simeq t_{3}$. The first example corresponds to the real sequence and the latter to the 'post-modulation' assumed above.

Calculating the intensity detected on a photodiode we get

$$
\begin{aligned}
& I_{\text {beat }}^{\mathrm{m}}(t) Z_{0}=\left|E_{\text {beat }}^{\mathrm{m}}(t)\right|^{2}=\text { const }+r \tau^{2} \alpha \epsilon I_{0} \\
& \quad \times\left[\mathrm{e}^{\mathrm{i} M \cos \left(\Omega_{\mathrm{m}} t\right)} \mathrm{e}^{\mathrm{i} \frac{4 \pi}{\lambda} a(t)}+\mathrm{e}^{-\mathrm{i} M \cos \left(\Omega_{\mathrm{m}} t\right)} \mathrm{e}^{-\mathrm{i} \frac{4 \pi}{\lambda} a(t)}\right]
\end{aligned}
$$

and after expanding the phase modulation term $\mathrm{e}^{\mathrm{i} M \cos \left(\Omega_{\mathrm{m}} t\right)}$ into a sum of harmonics of the modulation frequency $\Omega_{\mathrm{m}}$ this gives

$$
\begin{aligned}
& I_{\text {beat }}^{\mathrm{m}}(t) Z_{0}=\left|E_{\text {beat }}^{\mathrm{m}}(t)\right|^{2}=\text { const } \\
& \quad+r \tau^{2} \alpha \epsilon I_{0}\left[\sum_{l=-\infty}^{\infty} \mathrm{i}^{l} J_{l}(M) \mathrm{e}^{\mathrm{i} l \Omega_{\mathrm{m}} t} \mathrm{e}^{\mathrm{i} \frac{4 \pi}{\lambda} a(t)}\right. \\
& \left.\quad+\sum_{l=-\infty}^{\infty}(-\mathrm{i})^{l} J_{l}(M) \mathrm{e}^{-\mathrm{i} l \Omega_{\mathrm{m}} t} \mathrm{e}^{-\mathrm{i} \frac{4 \pi}{\lambda} a(t)}\right]
\end{aligned}
$$

with $J_{l}$ denoting the $l$ th-order Bessel function of the first kind. To simplify the formulae we disregard the constant term, which only describes the DC light power. This leads to the spectrum

$$
\begin{aligned}
& X^{\mathrm{m}}(\omega)=\frac{1}{\sqrt{2 \pi}} \int_{-\infty}^{\infty} I_{\text {beat }}(t) \mathrm{e}^{\mathrm{i} \omega t} \mathrm{~d} t \\
& =\frac{r \tau^{2} \alpha \epsilon}{\sqrt{2 \pi}} \int_{-\infty}^{\infty} I_{0}\left[\sum_{l=-\infty}^{\infty} \mathrm{i}^{l} J_{l}(M) \mathrm{e}^{\mathrm{i} l \Omega_{\mathrm{m}} t} \mathrm{e}^{\mathrm{i} \frac{4 \pi}{\lambda} a(t)}\right. \\
& \left.\quad+\sum_{l=-\infty}^{\infty}(-\mathrm{i})^{l} J_{l}(M) \mathrm{e}^{-\mathrm{i} l \Omega_{\mathrm{m}} t} \mathrm{e}^{-\mathrm{i} \frac{4 \pi}{\lambda} a(t)}\right] \mathrm{e}^{\mathrm{i} \omega t} \mathrm{~d} t \\
& =\frac{r \tau^{2} \alpha \epsilon I_{0}}{\sqrt{2 \pi}} \int_{-\infty}^{\infty}\left[\sum_{l=-\infty}^{\infty} \mathrm{i}^{l} J_{l}(M) \mathrm{e}^{\mathrm{i} l \Omega_{\mathrm{m}} t} \mathrm{e}^{\mathrm{i} \frac{4 \pi}{\lambda} a(t)}\right] \mathrm{e}^{\mathrm{i} \omega t} \mathrm{~d} t \\
& \quad+\frac{r \tau^{2} \alpha \epsilon I_{0}}{\sqrt{2 \pi}} \int_{-\infty}^{\infty}\left[\sum_{l=-\infty}^{\infty}(-\mathrm{i})^{l}\right. \\
& \left.\quad \times J_{l}(M) \mathrm{e}^{-\mathrm{i} l \Omega_{\mathrm{m}} t} \mathrm{e}^{-\mathrm{i} \frac{4 \pi}{\lambda} a(t)}\right] \mathrm{e}^{\mathrm{i} \omega t} \mathrm{~d} t .
\end{aligned}
$$

Exchanging the integration with the summation and taking time-independent terms out of the integration we get

$$
\begin{aligned}
& X^{\mathrm{m}}(\omega)=\sum_{l=-\infty}^{\infty} \mathrm{i}^{l} J_{l}(M) \frac{r \tau^{2} \alpha \epsilon I_{0}}{\sqrt{2 \pi}} \int_{-\infty}^{\infty} \mathrm{e}^{\mathrm{i} l \Omega_{\mathrm{m}} t} \mathrm{e}^{\mathrm{i} \frac{4 \pi}{\lambda} a(t)} \mathrm{e}^{\mathrm{i} \omega t} \mathrm{~d} t \\
& +\sum_{l=-\infty}^{\infty}(-\mathrm{i})^{l} J_{l}(M) \frac{r \tau^{2} \alpha \epsilon I_{0}}{\sqrt{2 \pi}} \int_{-\infty}^{\infty} \mathrm{e}^{-\mathrm{i} l \Omega_{\mathrm{m}} t} \mathrm{e}^{-\mathrm{i} \frac{4 \pi}{\lambda} a(t)} \mathrm{e}^{\mathrm{i} \omega t} \mathrm{~d} t .
\end{aligned}
$$

Comparing this with equation (5) we see that

$$
\begin{array}{r}
X^{\mathrm{m}}(\omega)=\sum_{l=-\infty}^{\infty} \mathrm{i}^{l} J_{l}(M) X^{+}\left(\omega+l \Omega_{\mathrm{m}}\right) \\
+\sum_{l=-\infty}^{\infty}(-\mathrm{i})^{l} J_{l}(M) X^{-}\left(\omega-l \Omega_{\mathrm{m}}\right) .
\end{array}
$$

The new spectrum consists of a series of multiples of the original spectrum components shifted in frequency by multiples of $\Omega_{\mathrm{m}}$ and scaled by the Bessel functions.

If we choose a modulation index (for a double pass through the phase shifter) of $M_{\mathrm{c}} \approx 2.408$ we get $J_{0}\left(M_{\mathrm{c}}\right) \approx 0$. Higher modulation indices where $J_{0}\left(M_{\mathrm{c}}\right) \approx 0$ would yield similar noise shifting out of the observation frequency band but are more difficult to achieve experimentally and yield no additional benefit. The first (lowest in frequency) spectrum component is then the one shifted by the modulation frequency. For this method, to rid the frequency range of interest of scattered light noise, the spectrum of the scattered light must be restricted to frequencies below the modulation frequency $\Omega_{\mathrm{m}}$. A simulation of this effect can be seen in figure 5 .

Modulating the phase or frequency of the input light as a method of reducing scattered light effects in interferometers has been mentioned in the literature before [8,9], but in recycled interferometers strong modulation of the injected light usually conflicts with the requirements of phase stability for field build-up in the recycling cavities.

\section{Experimental realization}

To shift the frequency of the scattered light as described above we need to modulate the phase of the beam in the scattering path. This can be achieved by modulating the optical path length between the scattering source and the interferometer by inserting an optical phase modulator. In many cases the beam diameter at this location prevents the use of electro-optical modulators exploiting the Pockels effect which would require very high electrical fields to achieve the critical modulation index $M_{\mathrm{c}}$. We propose instead to change the mechanical length of an optical element with an index of refraction $n \neq 1$ in the path by resonantly exciting a mechanical internal mode. In our case we chose a fused silica substrate with a diameter of $18 \mathrm{~cm}$ and a thickness of $10 \mathrm{~cm}$, i.e. a spare substrate of a GEO 600 main mirror. Gluing a piezoelectric actuator and a counterweight to the circumference of the substrate and suspending it in steel wire slings along the node lines of the desired mode allows us to modulate the thickness of the substrate. Additional pointed nylon bolts gently touching the circumference of the substrate on a node line avoid excess motion of the device at pendulum frequencies. For easing the (tilt or pitch) alignment around the horizontal axis perpendicular to the beam, the tension on the supporting wires could be adjusted.

Ideally the device would have AR coatings on both sides. In this experimental test set-up, we used an uncoated, fused silica blank and yet residual motion of the uncoated surfaces at frequencies in the observation band did not cause any harm. As the substrate is suspended, both surfaces will move by the same amount with respect to the centre of mass. To achieve the critical modulation $M_{\mathrm{c}}$ each surface of the substrate has to move with an amplitude $\delta l$ such that $2 \delta l(n-1) 22 \pi / \lambda=M_{\mathrm{c}}$ (the first factor of two coming from both surfaces moving and the second factor of two from the double pass) and the critical modulation is achieved. The light directly reflected from each surface then gets a phase shift of $M_{\mathrm{r}}=2 \delta l 2 \pi / \lambda$. In the case 


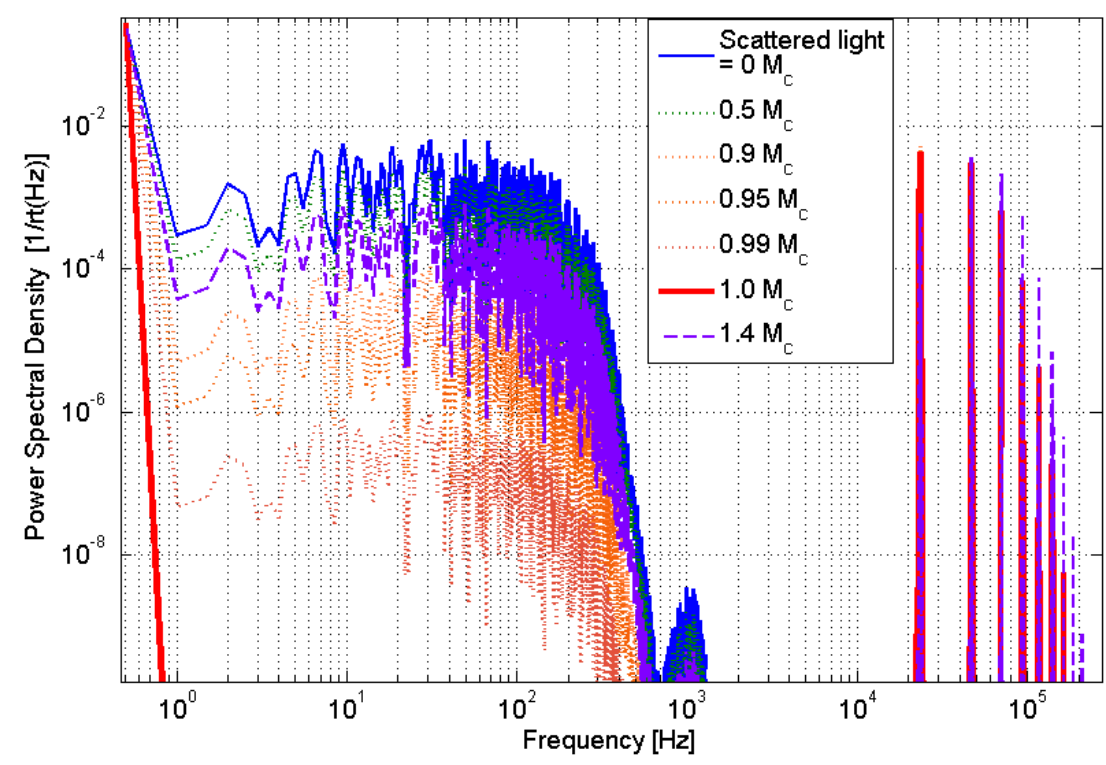

Figure 5. Simulated noise spectra with different levels of modulation. The modulation index, relative to the critical modulation index $M_{\mathrm{c}}$, is indicated in the legend. The uppermost, solid line (blue in the online version) is a signal without any modulation, i.e. the full scattering signal. The dotted curves (from green to dark orange in the online version and decreasing in amplitude) represent increasing modulation indices. The thick red curve that drops below the scale of the graph is the resulting noise level for critical modulation. It can be seen that, for the critical modulation, the signal content at the modulation frequency is highest. Increasing the modulation index beyond the critical modulation (shown by the dashed curve in purple) also increases the remaining scattered light contributions because we modulate beyond the zero crossing of the Bessel function $J_{0}$. The modulation frequency chosen for this example is $24 \mathrm{kHz}$. This agrees with the experimental set-up, where the frequency is determined by the mechanical properties of the substrate, and assures that the amplitude of the scattered light above half the modulation frequency is sufficiently low. Otherwise contributions being shifted downwards in frequency can spoil the result. We use simulated data here to show that the noise is not removed but shifted to higher frequencies. In the experimental demonstration the sampling rate of the data acquisition system used was too low to observe the upwardly shifted noise.

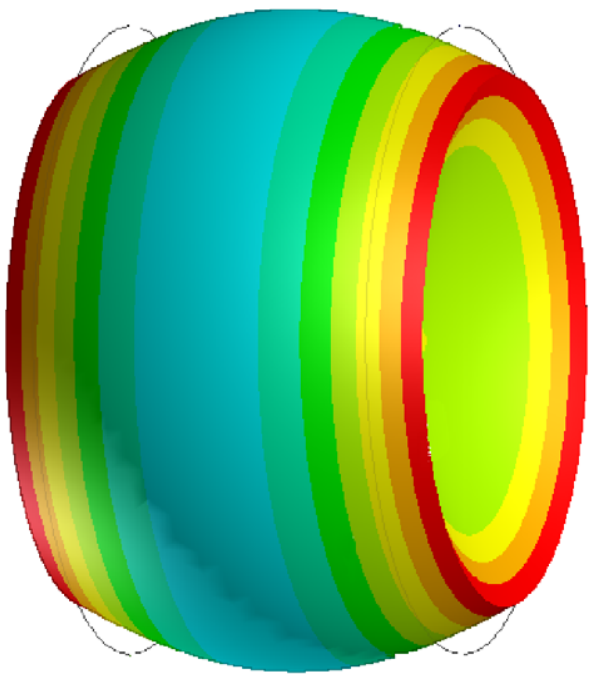

Figure 6. An illustration of the (exaggerated) deformation induced by the internal mode of the substrate used for phase shifting. It can be seen that the thickness of the substrate and therefore the optical path length changes. The dependence of the deformation on the distance from the axis can clearly be seen. If the critical modulation index is reached on axis it must therefore deviate from the critical value off axis.

when $n=1.5$ it follows that $M_{\mathrm{r}}=M_{\mathrm{c}}$. Hence as a coincidence (using fused silica which has a refractive index close to 1.5) modulating the thickness such that in a double pass the critical modulation index of $M_{\mathrm{c}} \approx 2.408$ is achieved, the part of the light reflected from the surfaces is also modulated critically and all the light reflected from the uncoated surfaces is also shifted to multiples of the modulation frequency. For very large beams which exceed the area where the condition of critical modulation is fulfilled and for transmitting as much light as possible an AR coating is still desirable. Figure 6 shows the mode of the substrate simulated with a built-in algorithm of the commercial software ANSYS and figure 7 shows a photograph of the set-up used in GEO 600. The same effect can be achieved by changing the geometrical path length as is shown in [7] for a slightly different purpose.

Often steering mirrors are implemented in the path to the scattering source. Placing one of these onto a piezoelectric actuator also allows us to modulate the phase of the returning beam and relaxes the restrictions in the modulation frequency used. Attention needs to be paid in this case that the front surface of the beam steerer does not get distorted too much, as the required modulation frequencies (to stay above the frequency of the main scattering noise contributions) may come close to or above the internal modes of the beam steerer. The advantage of the opto-mechanical device described here is its simple implementation into and removal from an existing set-up to check if backscattering from a particular port contributes to the system noise.

The opto-mechanical system was tested at one of the optical benches behind the folding mirror MFE in the east station of the GEO 600 detector. A schematic of the optical layout of GEO 600 is shown in figure 8. The mirror MFE 


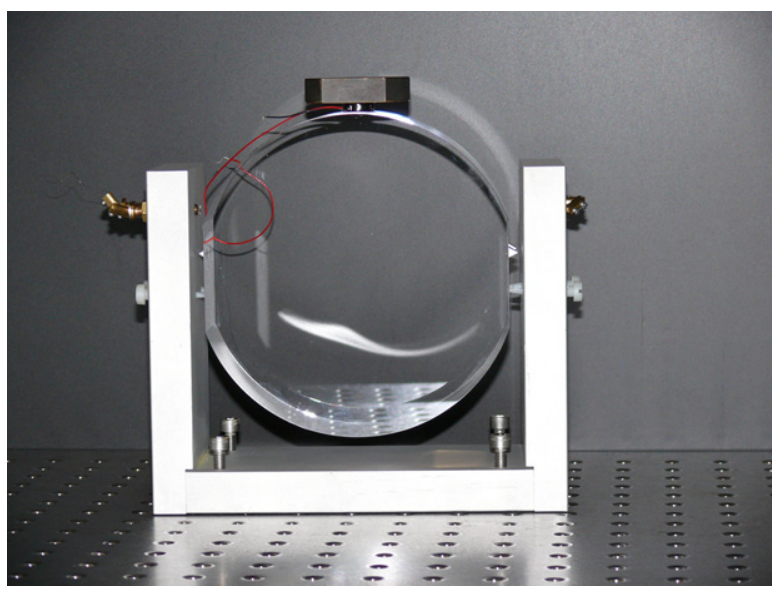

Figure 7. The opto-mechanical phase shifter is a fused silica substrate of $180 \mathrm{~mm}$ in diameter and $100 \mathrm{~mm}$ thickness suspended in two stainless steel wire loops. Four plastic screws lightly touching the sides of the optic at a node line of the desired eigenmode, i.e. the line on the surface that has no displacement for the mode considered, damped the pendulum motion of the optic in the wire loops. A piezoelectric actuator with a brass counterweight excites the resonance.

transmits about $10 \mathrm{ppm}$ of the incident beam, which at this position has a diameter of about $5 \mathrm{~cm}$. To have a clearly visible effect and to be able to test the suppression, we placed a loudspeaker with a metallic membrane as the scattering source in the focus of a lens behind MFE. The membrane of the loudspeaker was moved with an amplitude of about $1 \mathrm{~mm}$ and a frequency of $1 \mathrm{~Hz}$. This caused additional noise in the output of the detector and changed the green reference spectrum in figure 9 to the blue one. Applying the critical phase modulation shifted some of the additional noise to higher frequencies outside of the frequency range of the data acquisition system. The lowered noise floor is shown by the red trace. The fact

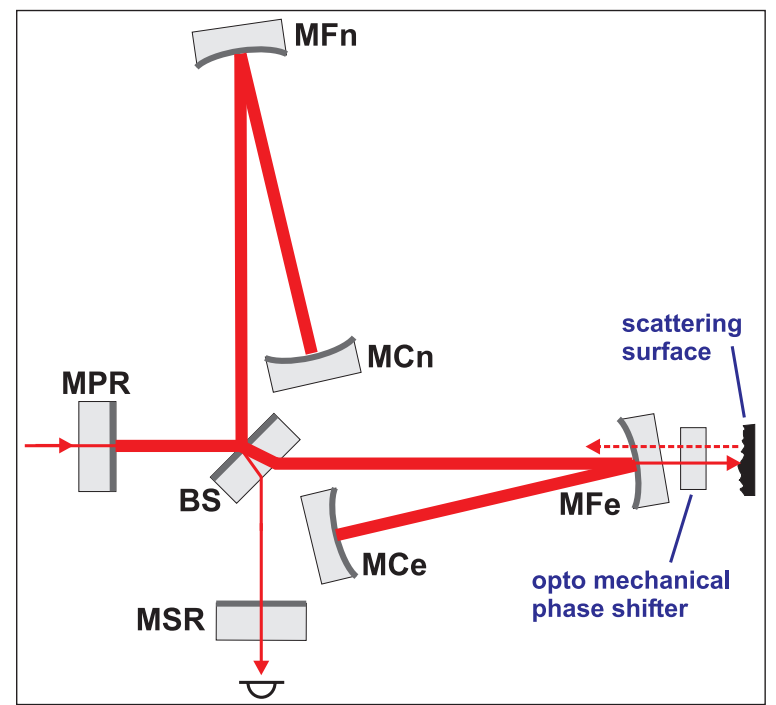

Figure 8. Optical layout of GEO 600 [4]. The light coming from the laser through the power recycling mirror (MPR) is split by the beamsplitter (BS) and travels along the two interferometer arms. Each $600 \mathrm{~m}$ arm is folded once at the far mirrors (MFn and MFe) and contains one of the end mirrors MCn and MCe. The signal recycling mirror (MSR) is placed in the output port. The scattering shifting experiment was placed behind the mirror MFe.

that the noise is not lowered to the reference level results from the critical modulation index condition not being fulfilled over the whole beam cross section. The amplitude of the oscillating substrate decreases towards the rim (see figure 6) and hence the outer parts of the beam fail to see the required critical phase modulation as in the inner parts. With a beam radius of $22 \mathrm{~mm}$, i.e. the beam radius at the mirror MFE, a simulation predicts a suppression factor of 9 which agrees well with the observed suppression of 10, i.e. within the known accuracy of the modulation level.

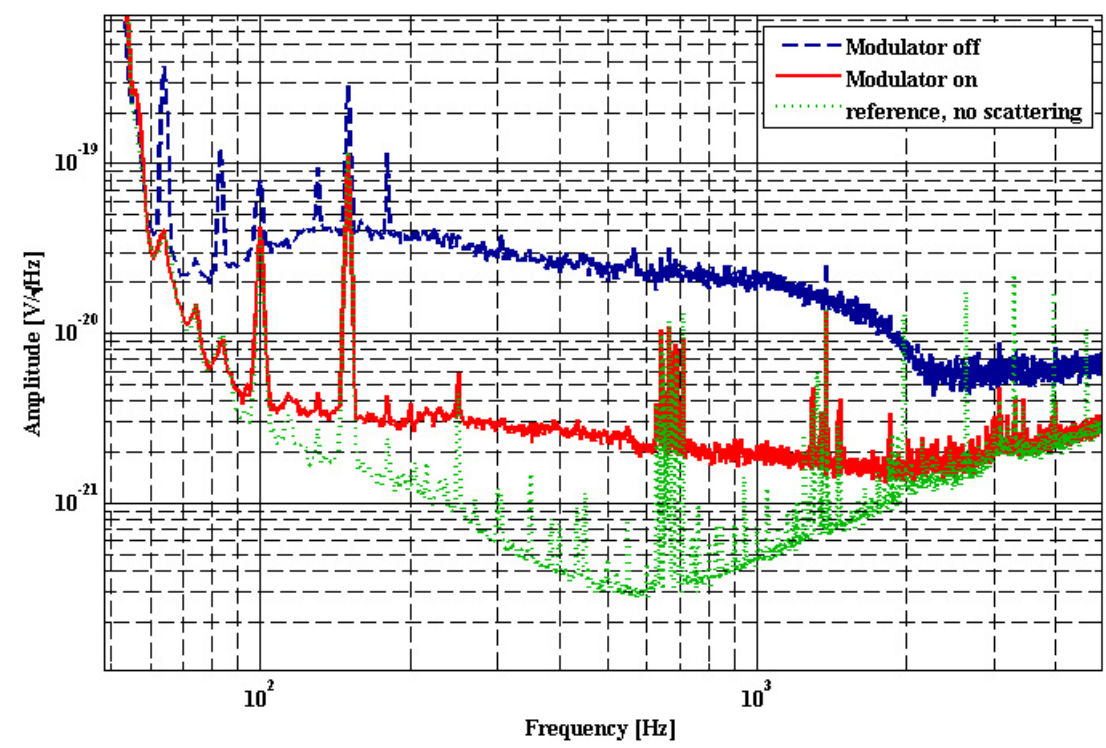

Figure 9. Effect of the phase modulation on the noise spectra of GEO 600. A reference noise spectrum without additional noise is shown in green. The blue trace is a sample of the noise spectrum with an additional scattering source placed behind MFE but without critical phase modulation. Switching on the phase modulation reduces the influence of the additional scattering to the red trace. 
If exact polarization preservation is important behind the modulator, the stress-induced birefringence needs to be taken into account. In the off-axis regions of the substrate we have a compression/stretching component in the direction perpendicular to the beam which will cause birefringence, i.e. will cause orthogonal polarizations to travel at different speeds and consequently locally change the polarization state of the beam. If the beam is small compared with the dimensions of the substrate (which is a prerequisite for good suppression anyway) this effect will be small. Commercial socalled photoelastic modulators exploit this effect in order to control the polarization of laser beams. An overview of the operational principle and some literature can be found in [6].

\section{Summary}

The opto-mechanical phase shifter discussed in this paper can easily be inserted into optical arrangements and in this way can help to localize surfaces that scatter light back into an optical device like a gravitational-wave detector. In cases where the scattering surface cannot be exchanged for one scattering less or shifted to a position where scattering has less adverse effects, e.g. radio-frequency photodetectors which need to be placed close to a small beam waist, the optomechanical phase shifter can be permanently built into the optical set-up. It has been tested in the main detection path of GEO 600 without any negative influence on the performance of the detector. The noise reduction of one order of magnitude could be demonstrated for a beam diameter of about $4.5 \mathrm{~cm}$.

\section{Acknowledgments}

We would like to thank Jan Harms for helpful discussions and Hung Ly and Stefan Gossler for the assembly of the phase shifter.

\section{References}

[1] Sigg D et al 2004 Commissioning of LIGO detectors Class. Quantum Grav. 21 S409-15

[2] Takahaschi R and (the TAMA Collaboration) 2004 Status of TAMA300 Class. Quantum Grav. 21 S403-8

[3] Acernese F et al 2004 Status of VIRGO Class. Quantum Grav. 21 S385-94

[4] Willke B 2004 Status of GEO 600 Class. Quantum Grav. 21 S417-23

[5] Hild S 2007 Beyond the first generation: extending the science range of the gravitational wave detector GEO $600 \mathrm{PhD}$ Thesis http://www.aei.mpg.de/pdf/doctoral/SHild_07.pdf

[6] http://www.hindsinstruments.com/PEM_Components/ techPapersMod.aspx

[7] Man C N et al 1978 Suppression of optical feedback effects on saturated absorption signals by phase modulation of the reflected light J. Phys. E: Sci. Instrum. 21 19-21

[8] Schilling R et al 1981 A method to blot out scattered light effects and its application to a gravitational wave detector J. Phys. E: Sci. Instrum. 14 65-70

[9] Beyersdorf P 2001 The polarization Sagnac interferometer for gravitational wave detection $P h D$ Thesis http://www.ligo. caltech.edu/docs/P/P010005-00.pdf 